\title{
On some physical aspects of isotropic cosmology in Riemann-Cartan spacetime
}

\author{
A.V. Minkevich, ${ }^{a, b}$ A.S. Garkun, ${ }^{a, c}$ and V.I. Kudin ${ }^{a, d}$ \\ ${ }^{a}$ Belarusian State University, Minsk, Belarus \\ ${ }^{b}$ Warmia and Mazury University in Olsztyn, Poland \\ ${ }^{c}$ The National Academy of Sciences of Belarus, Minsk, Belarus \\ ${ }^{d}$ Belarusian State Technical University, Minsk, Belarus \\ E-mail: minkav@bsu.by, awm@matman.uwm.edu.pl,garkun@bsu.by, \\ kudzin_w@tut.by
}

\begin{abstract}
Isotropic cosmology built in the framework of the Poincaré gauge theory of gravity based on sufficiently general expression of gravitational Lagrangian is considered. The derivation of cosmological equations and equations for torsion functions in the case of the most general homogeneous isotropic models is given. Physical aspects of isotropic cosmology connected with possible solution of dark energy problem and problem of cosmological singularity are discussed.
\end{abstract}

Keywords: Riemann-Cartan spacetime, isotropic cosmology, cosmological singularity, dark energy, dark matter 


\section{Contents}

1 Introduction 1

2 Principal relations of isotropic cosmology in Riemann-Cartan spacetime 2

3 Physical aspects of isotropic cosmology in Riemann-Cartan spacetime 5

4 Conclusion $\quad 11$

\section{Introduction}

Relativistic cosmology built on the base of the general relativity theory (GR) possesses some principal problems. The most principal problem of modern cosmology is the problem of invisible matter components in the Universe, which is connected with explanation of observational data: their explanation in the frame of GR leads to conclusion that about $96 \%$ energy in the Universe is connected with some hypothetical kinds of gravitating matter - dark energy and dark matter, and the energy of usual baryonic matter composes only about 4\%. As result the actual situation in cosmology and generally in gravitation theory is similar to that in physics at the beginning of XX century, when the notion of "ether" was introduced with the purpose to explain various electrodynamic phenomena. The creation of the special relativity theory by A. Einstein allowed to solve corresponding problems without "ether" notion. In addition as principal problem, which does not have acceptable solution still, remains the problem of the beginning of the Universe in time - the problem of cosmological singularity (PCS): different cosmological models built in the frame of GR and describing the evolution of the Universe have the beginning in time in the past, where singular state with divergent energy density and singular metrics appears. As it is known, the PCS is a particular case of general problem of GR - the problem of gravitational singularities.

Many attempts were undertaken with the purpose to solve indicated principal cosmological problems in the frame of GR and candidates to quantum gravitation theory - string theory/M-theory and loop quantum gravity as well as different generalizations of Einsteinian gravitation theory (see for example [1] and Refs herein). Radical ideas connected with notions of strings, extra-dimensions, space-time quantization etc are used in these works. Different hypothetical media and particles with unusual properties as possible candidates for dark energy and dark matter were introduced and discussed. Note that many existent generalizations of Einsteinian theory of gravitation do not have solid theoretical foundation.

At the same time now there is the gravitation theory built in the framework of common field-theoretical approach including the local gauge invariance principle, which is a natural generalization of GR and which offers opportunities to solve its principal problems. It is the Poincaré gauge theory of gravity (PGTG) - the gravitation theory in 4-dimensional physical space-time with the structure of Riemann-Cartan continuum $U_{4}$ [2-8]. The PGTG is a necessary generalization of metric theory of gravitation, if the Lorentz group is included into gauge group corresponding to gravitational interaction. In the frame of PGTG the energy momentum tensor together with the spin momentum of matter play the role of sources of gravitational field describing by means of space-time metrics and torsion. The simplest PGTG is Einstein-Cartan theory of gravity based on gravitational Lagrangian in the form 
of scalar curvature of $U_{4}$. Gravitational equations of this theory are identical to Einstein gravitational equations of GR in the case of spinless matter, and in the case of spinning sources Einstein-Cartan theory leads to linear relation between spacetime torsion and spin momentum of gravitating matter. Einstein-Cartan theory of gravity was investigated with the purpose to solve the PCS, some regular cosmological models filled with spinning Weyssenhoff fluid were built (see [9-11] and Refs herein). However, it appears that obtained results depend essentially on the type of spinning gravitating matter; so in the case of Dirac field as spinning matter source in Einstein-Cartan gravitational equations cosmological singularity generally speaking does not vanish (see for example $[12,13]$ ). ${ }^{1}$ Because of the fact that in the frame of Einstein-Cartan theory the torsion vanishes in absence of spin, the opinion that the torsion is generated by spin momentum of gravitating matter is widely held in literature. However, such situation seems unnatural, if we take into account that the torsion tensor plays the role of gravitational field strength corresponding to subgroup of spacetime translations connected directly in the frame of Noether formalism with energy-momentum tensor and consequently the torsion can be created by spinless matter [1]. The situation comes to normal by including to gravitational Lagrangian similarly to theory of Yang-Mills fields terms quadratic in gauge gravitational field strengths - the curvature and torsion tensors ${ }^{2}$. By using sufficiently general expression of gravitational Lagrangian including both a scalar curvature and various invariants quadratic in the curvature and torsion tensors with indefinite parameters, isotropic cosmology in the frame of PGTG was built and investigated in a number of papers (see [16-28] and Refs herein). As it was shown by investigation of homogeneous isotropic models (HIM), the gravitational interaction in the case of usual gravitating matter satisfying standard energy conditions by certain physical situations in the frame of PGTG is changed in comparison with GR and Newton's gravity theory and can be repulsive. As a result the PGTG offers opportunities to solve the PCS and problem of dark energy and possibly of dark matter by virtue of the change of gravitational interaction. These conclusions were obtained by investigation of HIM by using some restrictions on indefinite parameters of gravitational Lagrangian of PGTG.

The present paper is devoted to discussion of physical aspects of isotropic cosmology built in the frame of PGTG. At first the derivation of cosmological equations and equations for torsion functions in the case of sufficiently general expression of gravitational Lagrangian with indefinite parameters is given.

\section{Principal relations of isotropic cosmology in Riemann-Cartan spacetime}

In the framework of PGTG the role of gravitational field variables play the orthonormalized tetrad $h^{i}{ }_{\mu}$ and the Lorentz connection $A^{i k}{ }_{\mu}=-A^{k i}{ }_{\mu}$; corresponding field strengths are the torsion tensor $S^{i}{ }_{\mu \nu}$ and the curvature tensor $F^{i k}{ }_{\mu \nu}$ defined as

$$
\begin{gathered}
S_{\mu \nu}^{i}=\partial_{[\nu} h_{\mu]}^{i}-h_{k[\mu} A^{i k}{ }_{\nu]}, \\
F^{i k}{ }_{\mu \nu}=2 \partial_{[\mu} A_{\nu]}^{i k}+2 A_{[\mu}^{i l} A_{|l| \nu]}^{k},
\end{gathered}
$$

where holonomic and anholonomic space-time coordinates are denoted by means of greek and latin indices respectively.

\footnotetext{
${ }^{1}$ From physical point of view cosmological model with the jump of the Hubble parameter, in the frame of which the transition from compression stage to expansion stage happens instantly [14], hardly can be considered as a base of "nonsingular, big-bounce cosmology".

${ }^{2}$ For the first time it was shown in $[6,15]$.
} 
Because quadratic part of gravitational Lagrangian is unknown, we will consider the PGTG based on gravitational Lagrangian given in the following sufficiently general form corresponding to spacial parity conservation

$$
\begin{array}{r}
\mathcal{L}_{\mathrm{g}}=f_{0} F+F^{\alpha \beta \mu \nu}\left(f_{1} F_{\alpha \beta \mu \nu}+f_{2} F_{\alpha \mu \beta \nu}+f_{3} F_{\mu \nu \alpha \beta}\right)+F^{\mu \nu}\left(f_{4} F_{\mu \nu}+f_{5} F_{\nu \mu}\right) \\
+f_{6} F^{2}+S^{\alpha \mu \nu}\left(a_{1} S_{\alpha \mu \nu}+a_{2} S_{\nu \mu \alpha}\right)+a_{3} S^{\alpha}{ }_{\mu \alpha} S_{\beta}{ }^{\mu \beta}
\end{array}
$$

where $F_{\mu \nu}=F^{\alpha}{ }_{\mu \alpha \nu}, F=F^{\mu}{ }_{\mu}, f_{i}(i=1,2, \ldots, 6), a_{k}(k=1,2,3)$ are indefinite parameters, $f_{0}=(16 \pi G)^{-1}, G$ is Newton's gravitational constant (the light speed in the vacuum $c=1$ ). ${ }^{3}$ ${ }^{4}$ Gravitational equations of PGTG obtained from the action integral $I=\int\left(\mathcal{L}_{g}+\mathcal{L}_{m}\right) h d^{4} x$, where $h=\operatorname{det}\left(h^{i}{ }_{\mu}\right)$ and $\mathcal{L}_{m}$ is the Lagrangian of gravitating matter, contain the system of $16+24$ equations corresponding to gravitational variables $h^{i}{ }_{\mu}$ and $A^{i k}{ }_{\mu}$ :

$$
\begin{aligned}
& \nabla_{\nu} U_{i}{ }^{\mu \nu}+2 S^{k}{ }_{i \nu} U_{k}{ }^{\mu \nu}+2\left(f_{0}+2 f_{6} F\right) F^{\mu}{ }_{i}+4 f_{1} F_{k l i m} F^{k l \mu m}+4 f_{2} F^{k[m \mu] l} F_{k l i m} \\
&+4 f_{3} F^{\mu k l m} F_{l m i k}+2 f_{4}\left(F_{k i} F^{k \mu}+F^{\mu}{ }_{k i m} F^{k m}\right) \\
&+2 f_{5}\left(F_{k i} F^{\mu k}+F^{\mu}{ }_{k i m} F^{m k}\right)-h_{i}{ }^{\mu} \mathcal{L}_{g}=-T_{i}{ }^{\mu} \\
& 4 \nabla_{\nu}\left[\left(f_{0}+2 f_{6} F\right) h_{[i}{ }^{\nu} h_{k]}{ }^{\mu}+f_{1} F_{i k}{ }^{\nu \mu}+f_{2} F_{[i}{ }^{[\nu}{ }_{k]}{ }^{\mu]}+f_{3} F^{\nu \mu}{ }_{i k}\right. \\
&\left.+f_{4} F_{[k}{ }^{[\mu} h_{i]}{ }^{\nu]}+f_{5} F^{[\mu}{ }_{[k} h_{i]}{ }^{\nu]}\right]+U_{[i k]}^{\mu}=J_{[i k]}{ }^{\mu}
\end{aligned}
$$

where $U_{i}^{\mu \nu}=2\left(a_{1} S_{i}{ }^{\mu \nu}-a_{2} S^{[\mu \nu]}{ }_{i}-a_{3} S_{\alpha}{ }^{\alpha[\mu} h_{i}{ }^{\nu]}\right), T_{i}^{\mu}=-\frac{1}{h} \frac{\delta \mathcal{L}_{m}}{\delta h^{i} \mu}, J_{[i k]}^{\mu}=-\frac{1}{h} \frac{\delta \mathcal{L}_{m}}{\delta A^{i k_{\mu}}}, \nabla_{\nu}$ denotes the covariant operator having the structure of the covariant derivative defined in the case of tensor holonomic indices by means of Christoffel coefficients $\left\{\begin{array}{l}\lambda \\ \mu \nu\end{array}\right\}$ and in the case of tetrad tensor indices by means of anholonomic Lorentz connection $A^{i k}{ }_{\nu}$ (for example $\left.\nabla_{\nu} h_{\mu}^{i}=\partial_{\nu} h_{\mu}^{i}-\left\{\begin{array}{l}\lambda \\ \mu \nu\end{array}\right\} h_{\lambda}^{i}-A_{\nu}^{i k} h_{k \mu}\right)$. By using minimal coupling of gravitational field with matter the tensors $T_{i}^{\mu}$ and $J_{[i k]}^{\mu}$, which play the role of sources of gravitational field in equations (2.2)-(2.3), are the energy-momentum and spin momentum tensors of gravitating matter.

The structure of gravitational equations of PGTG (2.2)-(2.3) is simplified in the case of gravitating systems with high spacial symmetry, then the number of gravitational equations and their dependence on indefinite parameters are reduced. In accordance with cosmological principle in the frame of PGTG any HIM is described in general case by means of three functions of time: the scale factor of Robertson-Walker metrics $R(t)$ and two torsion functions $S_{1}(t)$ and $S_{2}(t)$ determining the following non-vanishing components of torsion tensor (with holonomic indices): $S^{1}{ }_{10}=S^{2}{ }_{20}=S^{3}{ }_{30}=S_{1}(t), S_{123}=S_{231}=S_{312}=S_{2}(t) \frac{R^{3} r^{2}}{\sqrt{1-k r^{2}}} \sin \theta$, where spatial spherical coordinates are used and $k=+1,0,-1$ for closed, flat and open models respectively $[32,33]$. The functions $S_{1}$ and $S_{2}$ have different properties with respect to spatial inversions, namely, unlike $S_{1}$ the function $S_{2}$ has pseudoscalar character. By using some tetrad (for example, in diagonal form) corresponding to Robertson-Walker metrics we

\footnotetext{
${ }^{3}$ It should be noted that one out of three parameters $f_{3}, f_{5}$ and $f_{6}$ can be excluded because of relation $\delta \int\left[F_{\mu \nu \alpha \beta} F^{\alpha \beta \mu \nu}-4 F_{\nu \mu} F^{\mu \nu}+F^{2}\right] h d^{4} x=0$.

${ }^{4}$ In general case without using condition of spacial parity conservation the gravitational Lagrangian can include a number invariants quadratic in the curvature and torsion tensors built by means of Levi-Civita discriminant tensor $\varepsilon_{\alpha \beta \mu \nu}$ [29-31]. In the frame of PGTG based on gravitational Lagrangian including also various invariants of type $\varepsilon F^{2}, \varepsilon S^{2}, \varepsilon^{2} F^{2}, \varepsilon^{2} S^{2}$ HIM were built and considered in [29].
} 
can calculate anholonomic Lorentz connection and the curvature tensor. Non-vanishing components of the curvature tensor for HIM are determined by means of the following functions $A_{k}(k=1,2,3,4)[19]$ :

$$
\begin{aligned}
A_{1}=\dot{H}+H^{2}-2 H S_{1}-2 \dot{S}_{1}, & A_{2}=\frac{k}{R^{2}}+\left(H-2 S_{1}\right)^{2}-S_{2}^{2}, \\
A_{3}=2\left(H-2 S_{1}\right) S_{2}, & A_{4}=\dot{S}_{2}+H S_{2},
\end{aligned}
$$

where $H=\dot{R} / R$ is the Hubble parameter and a dot denotes the differentiation with respect to time. As source of gravitational field in gravitational equations for HIM we will consider the average of distribution of gravitating matter describing by energy-momentum and spin momentum tensors. In accordance with cosmological principle the energy-momentum tensor is reduced to diagonal components determining the energy density $\rho$ and pressure $p$. In the case of non-polarized medium we put that the average of spin momentum tensor is equal to zero. Then the system of gravitational equations (2.2)-(2.3) for HIM is reduced to $2+2$ equations [19] given below:

$$
\begin{aligned}
a\left(H-S_{1}\right) S_{1}-2 b S_{2}^{2}-2 f_{0} A_{2}+4 f\left(A_{1}^{2}-A_{2}^{2}\right)+2 q_{2}\left(A_{3}^{2}-A_{4}^{2}\right) & =-\frac{\rho}{3}, \\
a\left(\dot{S}_{1}+2 H S_{1}-S_{1}^{2}\right)-2 b S_{2}^{2}-2 f_{0}\left(2 A_{1}+A_{2}\right)-4 f\left(A_{1}^{2}-A_{2}^{2}\right) & \\
-2 q_{2}\left(A_{3}^{2}-A_{4}^{2}\right) & =p, \\
f\left[\dot{A}_{1}+2 H\left(A_{1}-A_{2}\right)+4 S_{1} A_{2}\right]+q_{2} S_{2} A_{3}-q_{1} S_{2} A_{4}+\left(f_{0}+\frac{a}{8}\right) S_{1} & =0, \\
q_{2}\left[\dot{A}_{4}+2 H\left(A_{4}-A_{3}\right)+4 S_{1} A_{3}\right]-\left[4 f A_{2}+2 q_{1} A_{1}+\left(f_{0}-b\right)\right] S_{2} & =0 .
\end{aligned}
$$

The system of equations (2.5)-(2.8) includes the following five indefinite parameters:

$$
\begin{array}{r}
a=2 a_{1}+a_{2}+3 a_{3}, \quad b=a_{2}-a_{1}, \\
f=f_{1}+\frac{f_{2}}{2}+f_{3}+f_{4}+f_{5}+3 f_{6}, \\
q_{1}=f_{2}-2 f_{3}+f_{4}+f_{5}+6 f_{6}, \quad q_{2}=2 f_{1}-f_{2} .
\end{array}
$$

By analyzing the system of equations (2.5)-(2.8) we will use the Bianchi identities in the Riemann-Cartan continuum, which are reduced in the case of HIM to two following relations [19]:

$$
\begin{aligned}
& \dot{A}_{2}+2 H\left(A_{2}-A_{1}\right)+4 S_{1} A_{1}+2 S_{2} A_{4}=0, \\
& \dot{A}_{3}+2 H\left(A_{3}-A_{4}\right)+4 S_{1} A_{4}-2 S_{2} A_{1}=0 .
\end{aligned}
$$

The system of gravitational equations (2.5)-(2.8) allows to obtain the generalization of Friedmann cosmological equations for HIM, and also equations for the torsion functions $S_{1}$ and $S_{2}$. By adding eqs. (2.5)-(2.6) and using the definition (2.4) of curvature functions $A_{1}$ and $A_{2}$, we find the expression for scalar curvature $F=6\left(A_{1}+A_{2}\right)$ :

$$
F=\frac{1}{2\left(f_{0}+a / 8\right)}\left[\rho-3 p-12(b+a / 8) S_{2}^{2}+\frac{3 a}{2}\left(\frac{k}{R^{2}}+\dot{H}+2 H^{2}\right)\right] .
$$


Then the equation (2.5) together with (2.11) allow to find the following expressions for curvature functions $A_{1}$ and $A_{2}$ :

$$
\begin{aligned}
A_{1}= & -\frac{1}{12\left(f_{0}+a / 8\right) Z}\left\{\rho+3 p-\frac{2 f}{3} F^{2}+8 q_{2} F S_{2}^{2}\right. \\
& \left.-12 q_{2}\left[\left(H S_{2}+\dot{S}_{2}\right)^{2}+4\left(\frac{k}{R^{2}}-S_{2}^{2}\right) S_{2}^{2}\right]-\frac{3 a}{2}\left(\dot{H}+H^{2}\right)\right\}, \\
A_{2}= & \frac{1}{6\left(f_{0}+a / 8\right) Z}\left\{\rho-6(b+a / 8) S_{2}^{2}+\frac{f}{3} F^{2}+\frac{3 a}{4}\left(\frac{k}{R^{2}}+H^{2}\right)\right. \\
& \left.-6 q_{2}\left[\left(H S_{2}+\dot{S}_{2}\right)^{2}+4\left(\frac{k}{R^{2}}-S_{2}^{2}\right) S_{2}^{2}\right]\right\},
\end{aligned}
$$

where $Z=1+\frac{1}{\left(f_{0}+a / 8\right)}\left(\frac{2 f}{3} F-4 q_{2} S_{2}^{2}\right)$. The gravitational equation (2.7) together with Bianchi identity (2.9) by using the definition (2.4) of the curvature functions $A_{3}$ and $A_{4}$ and the formulae (2.11) for scalar curvature $F$ allow to obtain the following expression for torsion function $S_{1}$ :

$$
S_{1}=-\frac{1}{6\left(f_{0}+a / 8\right) Z}\left[f \dot{F}+6\left(2 f-q_{1}+2 q_{2}\right) H S_{2}^{2}+6\left(2 f-q_{1}\right) S_{2} \dot{S}_{2}\right] .
$$

By using the Bianchi identity (2.10) together with (2.4) and (2.11) we find from gravitational equation (2.8) the following differential equation of the second order for torsion function $S_{2}$ :

$$
\begin{aligned}
q_{2} & {\left[\ddot{S}_{2}+3 H \dot{S}_{2}+\left(3 \dot{H}-4 \dot{S}_{1}+4 S_{1}\left(3 H-4 S_{1}\right)\right) S_{2}\right] } \\
& -\left[\frac{q_{1}+q_{2}}{3} F+\left(f_{0}-b\right)-2\left(q_{1}+q_{2}-2 f\right) A_{2}\right] S_{2}=0 .
\end{aligned}
$$

The generalization of Friedmann cosmological equations for HIM we obtain by substituting into definitions (2.4) of curvature functions $A_{1}$ and $A_{2}$ their expressions (2.12). These equations contain the torsion functions $S_{1}$ and $S_{2}$ with their first derivatives, which are determined by equations (2.13) and (2.14). So far, we have not used any restrictions on indefinite parameters of $\mathcal{L}_{\mathrm{g}}$. From formulas (2.11) and (2.13) for scalar curvature $F$ and torsion function $S_{1}$ we see that cosmological equations do not contain higher derivatives of the scale factor $R$ only if $a=0$ (see $[15,24]$ ). With the purpose to exclude higher derivatives of $R$ from cosmological equations the restriction $a=0$ was used in our works. It should be noted that isotropic cosmology with $a \neq 0$ possesses some principal problems [34].

\section{Physical aspects of isotropic cosmology in Riemann-Cartan spacetime}

By putting $a=0$ we write the cosmological equations for HIM in the following form:

$$
\begin{aligned}
\frac{k}{R^{2}}+ & \left(H-2 S_{1}\right)^{2}=\frac{1}{6 f_{0} Z}\left[\rho+6\left(f_{0} Z-b\right) S_{2}^{2}+\frac{\alpha}{4}\left(\rho-3 p-12 b S_{2}^{2}\right)^{2}\right] \\
& -\frac{3 \alpha \varepsilon f_{0}}{Z}\left[\left(H S_{2}+\dot{S}_{2}\right)^{2}+4\left(\frac{k}{R^{2}}-S_{2}^{2}\right) S_{2}^{2}\right], \\
\dot{H}+ & H^{2}-2 H S_{1}-2 \dot{S}_{1}=-\frac{1}{12 f_{0} Z}\left[\rho+3 p-\frac{\alpha}{2}\left(\rho-3 p-12 b S_{2}^{2}\right)^{2}\right] \\
& -\frac{\alpha \varepsilon}{Z}\left(\rho-3 p-12 b S_{2}^{2}\right) S_{2}^{2}+\frac{3 \alpha \varepsilon f_{0}}{Z}\left[\left(H S_{2}+\dot{S}_{2}\right)^{2}+4\left(\frac{k}{R^{2}}-S_{2}^{2}\right) S_{2}^{2}\right],
\end{aligned}
$$


where the following notations of indefinite parameters are introduced - the parameter $\alpha=\frac{f}{3 f_{0}^{2}}$ with inverse dimension of energy density $(f>0)$ and dimensionless parameter $\varepsilon=q_{2} / f$; the scalar curvature is $F=\frac{1}{2 f_{0}}\left(\rho-3 p-12 b S_{2}^{2}\right)$ and $Z=1+\alpha\left(\rho-3 p-12\left(b+\varepsilon f_{0}\right) S_{2}^{2}\right)$. According to (2.13)-(2.14) the torsion functions are determined by equations:

$$
\begin{aligned}
& S_{1}=-\frac{\alpha}{4 Z}\left[\dot{\rho}-3 \dot{p}+12 f_{0}(3 \varepsilon+\omega) H S_{2}^{2}-12\left(2 b-(\varepsilon+\omega) f_{0}\right) S_{2} \dot{S}_{2}\right], \\
& \varepsilon\left[\ddot{S}_{2}+3 H \dot{S}_{2}+\left(3 \dot{H}-4 \dot{S}_{1}+12 H S_{1}-16 S_{1}^{2}\right) S_{2}\right] \\
& -\frac{1}{3 f_{0}}\left[\left(1-\frac{1}{2} \omega\right)\left(\rho-3 p-12 b S_{2}^{2}\right)+\frac{\left(1-b / f_{0}\right)}{\alpha}+6 f_{0} \omega A_{2}\right] S_{2}=0,
\end{aligned}
$$

where dimensionless parameter $\omega=\frac{2 f-q_{1}-q_{2}}{f}$ is introduced. By given equation of state for gravitating matter cosmological equations (3.1)-(3.2) together with equations (3.3)-(3.4) for torsion functions describe the evolution of HIM (without higher derivatives) in the frame of PGTG. The equations (3.1)-(3.4) of isotropic cosmology obtained in the frame of PGTG based on gravitational Lagrangian (2.1) contain in general case four indefinite parameters: $\alpha$ (or $f$ ), $b, \varepsilon$ and $\omega$. These parameters have certain values by supposing that the PGTG is correct gravitation theory. We can find restrictions on indefinite parameters by analyzing physical consequences of isotropic cosmology in dependence on values of indefinite parameters, by which these consequences are the most satisfactory and correspond to observational cosmological data.

a) Acceleration of cosmological expansion at present epoch as gravitational vacuum effect

At first we will consider the behaviour of cosmological solutions at asymptotics, where energy density is sufficiently small. It is easy to show that the cosmological equations at asymptotics take the form of Friedmann cosmological equations of GR with effective cosmological constant if dimensionless parameters $\varepsilon$ and $\omega$ are sufficiently small: $|\varepsilon|<<1,|\omega|<<1$ (see $[19,21,24]$ ). In fact by supposing that the torsion function $S_{2}$ does not vanish we obtain from (3.4) in this case in the lowest approximation with respect to small parameters $\varepsilon$ and $\omega$ the following expression for $S_{2}$ :

$$
S_{2}^{2}=\frac{1}{12 b}\left[\rho-3 p+\frac{1-b / f_{0}}{\alpha}\right] .
$$

Because in considered approximation $Z \rightarrow\left(b / f_{0}\right), S_{1} \rightarrow 0$, the cosmological equations (3.1)(3.2) take the following form:

$$
\begin{gathered}
\frac{k}{R^{2}}+H^{2}=\frac{1}{6 f_{0}}\left[\rho \frac{f_{0}}{b}+\frac{1}{4 \alpha}\left(1-\frac{b}{f_{0}}\right)^{2} \frac{f_{0}}{b}\right], \\
\dot{H}+H^{2}=-\frac{1}{12 f_{0}}\left[(\rho+3 p) \frac{f_{0}}{b}-\frac{1}{2 \alpha}\left(1-\frac{b}{f_{0}}\right)^{2} \frac{f_{0}}{b}\right] .
\end{gathered}
$$

Note that effective cosmological constant in cosmological equations (3.6)-(3.7) appears by virtue of the presence of the constant term in expression (3.5) for the torsion function $S_{2}$. The cosmological equations (3.6)-(3.7) contain two indefinite parameters: $\alpha$ and $b$; the restrictions on these parameters can be obtained by using cosmological data and by comparing eqs. (3.6)(3.7) with Friedmann cosmological equations:

$$
\frac{k}{R^{2}}+H^{2}=\frac{1}{6 f_{0}} \rho_{t o t}
$$




$$
\dot{H}+H^{2}=-\frac{1}{12 f_{0}}\left(\rho_{t o t}+3 p_{t o t}\right)
$$

where $\rho_{t o t}$ and $p_{t o t}$ are total values of energy density and pressure including contributions of three components - baryonic matter, dark matter and dark energy: $\rho_{t o t}=\rho_{B M}+\rho_{D M}+$ $\rho_{D E}, p_{t o t}=p_{B M}+p_{D M}+p_{D E}$. In the case of standard $\Lambda C D M$-model $(k=0)$ one uses at present epoch for baryonic and dark matter the equation of state of dust $\left(p_{B M}=p_{D M}=0\right)$, and for dark energy $p_{D E}=-\rho_{D E}$. According to observational data, the Universe evolution approximately is in agreement with cosmological equations (3.8)-(3.9) for $\Lambda C D M$-model, if one supposes that the contribution of baryonic matter, dark matter and dark energy to energy density of the Universe is the following: $\rho_{B M 0}=0.04 \rho_{c r}, \rho_{D M 0}=0.23 \rho_{c r}, \rho_{D E 0}=0.73 \rho_{c r}$, where $\rho_{c r}=6 f_{0} H_{0}^{2}$ and values of physical parameters at present epoch are denoted by means of the index "0". The cosmological equations (3.6)-(3.7) lead to the same consequences as equations of standard $\Lambda C D M$-model if one supposes that the second term inside the parentheses in eq. (3.6) is equal to $\rho_{D E 0}$ and the first term $\rho \frac{f_{0}}{b}$ is equal to the sum of energy density of baryonic and dark matter. The first supposition leads to the relation for indefinite parameters $\alpha$ and $b$, and the second supposition gives the dependence of parameter $b$ on the presence of the dark matter and its contribution to the energy density $\rho$. By using approximative estimation of contribution of baryonic and dark matter to the energy density in the frame of $\Lambda C D M$-model given above we obtain: $\frac{4}{27} f_{0} \leq b<f_{0}$. The lower estimation of $b$ is valid, if the dark matter practically does not exist [21]. If dark matter exists in accordance with $\Lambda C D M$-model, the value of $b$ is very near to $f_{0}$ being less than $f_{0}$. By taking into account the role of dark matter in galaxies and their accumulations in the frame of GR, we have to conclude that the investigation of dark matter problem in the frame of PGTG assumes the study of inhomogeneous gravitating systems at astrophysical scale.

It should be noted that in the case of discussed spatially flat HIM the spacetime in the vacuum has the structure of de Sitter spacetime with non-vanishing torsion (but not Minkowski spacetime) [23] that ensures the accelerating cosmological expansion at present epoch. Dynamical properties of gravitating vacuum in the frame of PGTG appear without introducing of cosmological constant and are connected essentially with spacetime torsion.

b) Limiting energy density and gravitational repulsion at extreme conditions

Unlike the asymptotics of cosmological solutions, where the effect of gravitational repulsion leading to acceleration of cosmological expansion at present epoch is provoked by pseudoscalar torsion function $S_{2}$, at extreme conditions at the beginning of cosmological expansion the effect of gravitational repulsion is connected essentially also with the torsion function $S_{1}$. By certain restrictions on indefinite parameters cosmological equations for HIM filled with usual gravitating matter satisfying energy dominance conditions lead to existence of limiting (maximum) energy density, near to which the gravitational interaction is repulsive that ensures the regularization of cosmological solutions of such models in the frame of PGTG. At the first time the conclusion about possible existence of limiting energy density was obtained in the case of HIM with the only torsion function $S_{1}\left(S_{2}=0\right)$ [15] (see also [16]). However, because the vacuum in such models has the structure of Minkowski spacetime [23], their behaviour at asymptotics does not allow to explain observable accelerating cosmological expansion at present epoch. Simultaneous solution of PCS and dark energy problem can be obtained in the case of HIM with two torsion functions. The existence of limiting energy density follows strictly from eqs. (3.1)-(3.4), if $\varepsilon=0$ [24], that leads to their 
essential simplification. Cosmological equations (3.1)-(3.4) take the following form:

$$
\begin{aligned}
& \frac{k}{R^{2}}+\left(H-2 S_{1}\right)^{2}-S_{2}^{2}=\frac{1}{6 f_{0} Z}\left[\rho-6 b S_{2}^{2}+\frac{\alpha}{4}\left(\rho-3 p-12 b S_{2}^{2}\right)^{2}\right], \\
& \dot{H}+H^{2}-2 H S_{1}-2 \dot{S}_{1}=-\frac{1}{12 f_{0} Z}\left[\rho+3 p-\frac{\alpha}{2}\left(\rho-3 p-12 b S_{2}^{2}\right)^{2}\right],
\end{aligned}
$$

where $Z=1+\alpha\left(\rho-3 p-12 b S_{2}^{2}\right)$. The torsion function $S_{1}$ determined by (3.3) and the torsion function $S_{2}^{2}$ obtained from (3.4) at $\varepsilon=0$ take the form [24]:

$$
\begin{aligned}
& S_{1}=-\frac{\alpha}{4 Z}\left[\dot{\rho}-3 \dot{p}+12 f_{0} \omega H S_{2}^{2}-12\left(2 b-\omega f_{0}\right) S_{2} \dot{S}_{2}\right], \\
& S_{2}^{2}=\frac{\rho-3 p}{12 b}+\frac{1-\left(b / 2 f_{0}\right)(1+\sqrt{X})}{12 b \alpha(1-\omega / 4)},
\end{aligned}
$$

where $X=1+\omega\left(f_{0}^{2} / b^{2}\right)\left[1-\left(b / f_{0}\right)-2(1-\omega / 4) \alpha(\rho+3 p)\right]$. If parameters $\alpha$ and $\omega$ are positive, the condition $X \geq 0$ restricts admissible values of energy density and pressure and by taking into account smallness of $\omega$ can be written in the following form:

$$
X=1-2\left(f_{0}^{2} / b^{2}\right) \omega \alpha(\rho+3 p) \geq 0 .
$$

In the case of models filled with usual matter minimally coupled with gravitation with energy density $\rho_{m}>0$ and pressure $p_{m}=p_{m}\left(\rho_{m}\right) \geq 0$, for which

$$
\dot{\rho}_{m}+3 H\left(\rho_{m}+p_{m}\right)=0
$$

the equality (3.14) determines a limiting (maximum) energy density $\rho_{\max }$. When energy density $\rho_{m}$ is comparable with $\rho_{\max }$, the gravitational interaction has the character of repulsion ensuring the regularity of such systems. The order of $\rho_{\max }$ is determined by the value of $(\omega \alpha)^{-1}$. In the frame of classical theory the value of $\rho_{\max }$ has to be less than the Planck energy density. The state with $\rho_{m}=\rho_{\max }$ corresponds to a bounce and near to this state in linear approximation with respect to $\sqrt{X}$ the behaviour of the Hubble parameter and its time derivative are determined according to (3.10)-(3.13) and (3.15) by the following way [24]:

$$
\begin{aligned}
& H_{ \pm}= \pm \frac{2 b^{2}}{3 f_{0}^{2} \omega \alpha} \frac{\sqrt{X}\left[(1 / 4 b)\left(\rho_{m}+p_{m}\right)-\left(k / R^{2}\right)\right]^{1 / 2}}{\left(3 \frac{d p_{m}}{d \rho_{m}}+1\right)\left(\rho_{m}+p_{m}\right)} \\
& \dot{H}=\frac{4 b^{2}}{3 f_{0}^{2} \omega \alpha} \frac{(1 / 4 b)\left(\rho_{m}+p_{m}\right)-\left(k / R^{2}\right)}{\left(3 \frac{d p_{m}}{d \rho_{m}}+1\right)\left(\rho_{m}+p_{m}\right)}
\end{aligned}
$$

The solution $H_{-}$corresponds to the compression stage before a bounce, and the solution $H_{+}$ corresponds to the expansion stage after a bounce. In accordance with (3.16) the evolution of scale factor $R(t)$ near a bounce takes the following form: $R(t)=R_{\min }+r_{1} t^{2}+\ldots$, where $t=0$ corresponds to a bounce, $R_{\min }$ is minimum value of $R$ depending on limiting energy density and given equation of state, the value of $r_{1}>0$ is expressed by $\dot{H}$ at a bounce. It should be noted that in accordance with expression (3.13) the condition $S_{2}^{2}>0$ will be fulfilled, if equation of state of gravitating matter at the beginning of cosmological expansion satisfies the following condition $p_{m} \leq \rho_{m} / 3$. All cosmological solutions are regular with respect to energy density, the scale factor $R$ and the Hubble parameter $H$ by virtue of existence of limiting 
energy density. Unlike HIM with the only torsion function $S_{1}$, in the case of considered HIM with two torsion functions the torsion does not diverge by reaching limiting energy density. Physical results connected with limiting energy density were obtained by using the condition $\varepsilon=0$. If the parameter $\varepsilon$ does not vanish and is sufficiently small, these results remain valid in the lowest approximation with respect to $\varepsilon$ and will be corrected in further approximations.

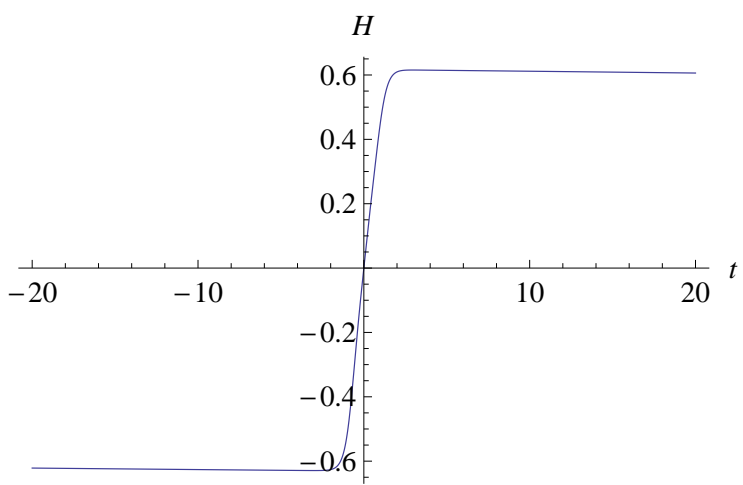

Figure 1. The behaviour of $H$ during the transition stage.

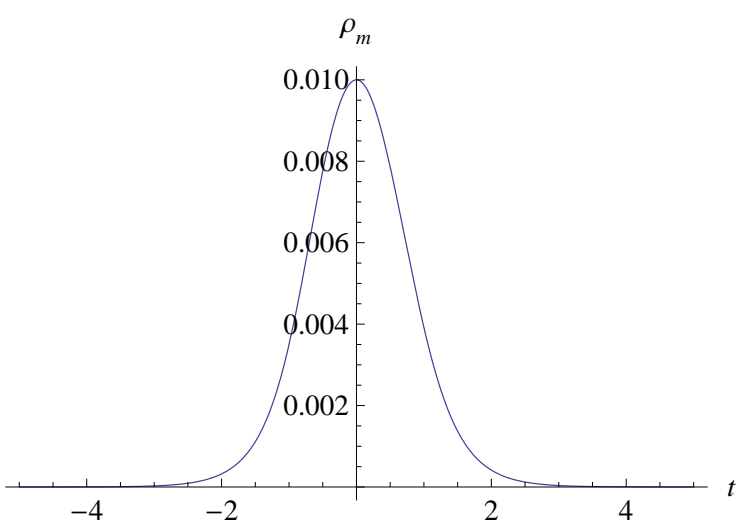

Figure 3. The behaviour of $\rho_{m}$ during the transition stage.

c) About regular inflationary cosmology

Equations (3.1)-(3.4) for HIM allow to build in the frame of PGTG regular inflationary cosmology by including at initial stage of cosmological expansion besides usual gravitating matter with energy density $\rho_{m}>0$ and pressure $p_{m}=p_{m}\left(\rho_{m}\right)$ also some scalar field $\phi$ with potential $V=V(\phi)$ as component of gravitating matter. By minimal coupling with gravitation the equation for scalar field takes the usual form as in GR:

$$
\ddot{\phi}+3 H \dot{\phi}=-\frac{\partial V}{\partial \phi} .
$$




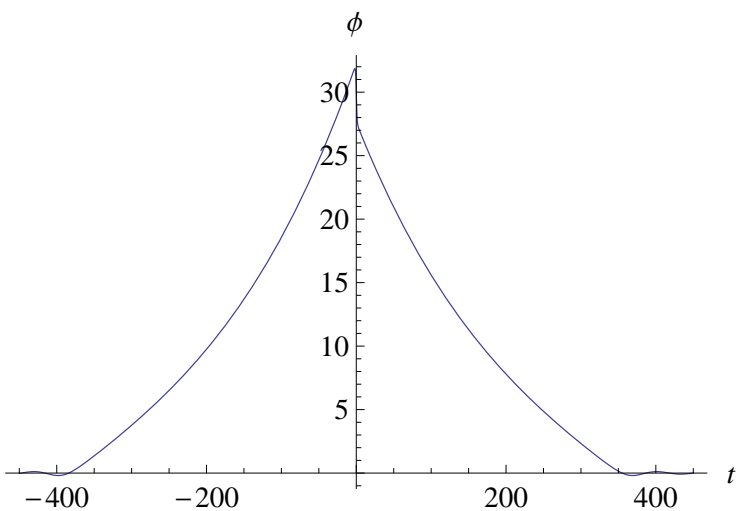

Figure 5. The behaviour of $\phi$ during the inflationary stage.

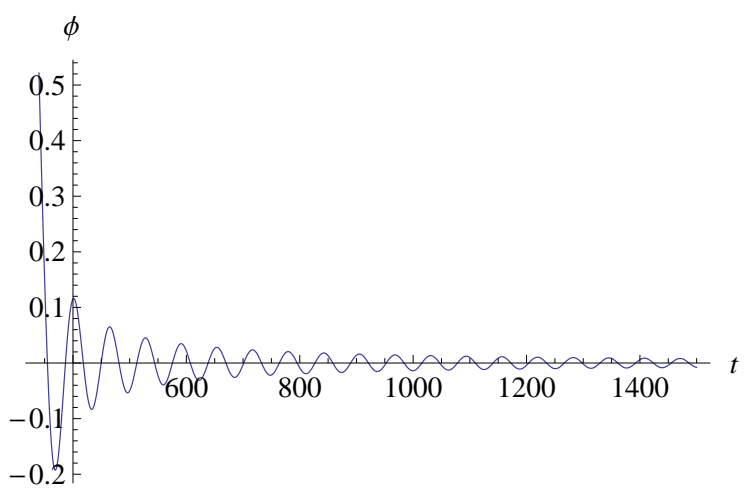

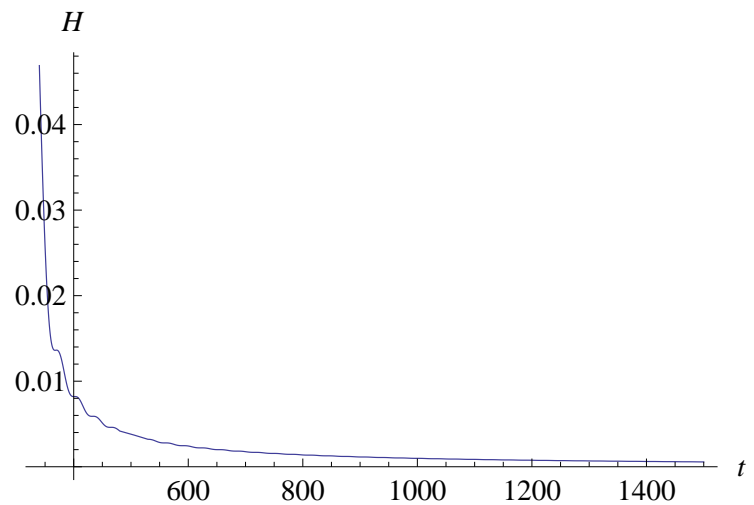

Figure 6. The behaviour of $H$ during the postinflationary stage.

Figure 7. The behaviour of $\phi$ during the postinflationary stage.

Then the total energy density $\rho$ and pressure $p$ are the following:

$$
\rho=\frac{1}{2} \dot{\phi}^{2}+V+\rho_{m} \quad(\rho>0), \quad p=\frac{1}{2} \dot{\phi}^{2}-V+p_{m} .
$$

We will consider the simplest case $\varepsilon=0$. Then the condition $X \geq 0$ determines in space of matter parameters $\left(\rho_{m}, \phi, \dot{\phi}\right)$ domain of their admissible values. This domain is limited by surface $L$ defined as $X=0$. The Hubble parameter does not vanish by reaching a limiting surface $L$ and according to (3.10) by taking into account (3.12)-(3.15) its value on surface $L$ is:

$$
H_{L}=\frac{-2 \frac{\partial V}{\partial \phi} \dot{\phi}}{\left(3 \frac{d p_{m}}{d \rho_{m}}+1\right)\left(\rho_{m}+p_{m}\right)+4 \dot{\phi}^{2}} .
$$

The bounce in this case takes place in points of extremum surface in space of matter parameters $\left(\rho_{m}, \phi, \dot{\phi}\right)$, equation of which we obtain by setting $H=0$ in cosmological equation (3.10). 
Cosmological solutions can be found by numerical integration of eqs. (3.11), (3.15) and (3.17) by taking into account (3.12)-(3.13) and by choosing initial conditions on extremum surface. Preliminarily these equations have to be transformed to dimensionless form by using the following dimensionless quantities:

$$
\begin{aligned}
t \rightarrow \tilde{t}=t / \sqrt{6 f_{0} \omega \alpha}, & R \rightarrow \tilde{R}=R / \sqrt{6 f_{0} \omega \alpha}, \\
\rho \rightarrow \tilde{\rho}=\omega \alpha \rho, & p \rightarrow \tilde{p}=\omega \alpha p, \\
\phi \rightarrow \tilde{\phi}=\phi / \sqrt{6 f_{0}}, & b \rightarrow \tilde{b}=b / f_{0}, \\
H \rightarrow \tilde{H}=\tilde{R}^{\prime} / R=H \sqrt{6 f_{0} \omega \alpha}, & V \rightarrow \tilde{V}=\omega \alpha V, \\
S_{1,2} \rightarrow \tilde{S}_{1,2}=S_{1,2} \sqrt{6 f_{0} \omega \alpha}, &
\end{aligned}
$$

where the differentiation with respect to dimensionless time $\tilde{t}$ is denoted by means of the prime.

Similarly to inflationary cosmological models with the only torsion function (see $[16$, 18]), if initial value of scalar field is sufficiently large, regular cosmological solution contains transition stage from compression to expansion, inflationary stage with slow-rolling behaviour of scalar field and post-inflationary stage with oscillating scalar field. Corresponding inflationary cosmological models have bouncing character and are totally regular $[27,28]$ : the regularity takes place not only with respect to energy density, metrics and Hubble parameter, but also with respect to torsion and curvature functions.

As illustration numerical inflationary solution in the case of flat model $(k=0)$ for the Hubble parameter and scalar field obtained by choosing quadratic scalar field potential $V=m^{2} \phi^{2} / 2\left(\tilde{V}=\tilde{m}^{2} \tilde{\phi}^{2} / 2, \tilde{m}=m \sqrt{6 f_{0} \omega \alpha}\right)$ and $p_{\mathrm{m}}=\rho_{\mathrm{m}} / 3$ is given in Fig. 1-7 (the tildes in figures are omitted). Numerical solution was obtained by choosing the following values of indefinite parameters and initial conditions on extremum surface $H=0: \tilde{b}=0.999, \tilde{\omega}=10^{-8}$, $\tilde{m}=0.1, \tilde{\phi}_{0}=30, \tilde{\phi}_{0}^{\prime}=-2.14, \tilde{\rho}_{m 0}=0.01$.

\section{Conclusion}

The investigation of isotropic cosmology built in the framework of PGTG shows that this theory of gravity offers opportunities to solve some principal problems of GR. It is achieved by virtue of the change of gravitational interaction by certain physical conditions in the frame of PGTG in comparison with GR. The change of gravitational interaction is provoked by more complicated structure of physical spacetime, namely by spacetime torsion. The further study of isotropic cosmology will allow to make more precise indefinite parameters of gravitational Lagrangian of PGTG, and the investigation of inhomogeneous and anisotropic gravitating systems will show to what extent physical results obtained in the frame of isotropic cosmology are valid.

\section{References}

[1] A.V. Minkevich, Gravitational interaction and Poincaré gauge theory of gravity, Acta Physica Polonica B , 40 (2009) 229 [arXiv:0808.0239].

[2] T.W.B. Kibble, Lorentz invariance and the gravitational field, Journal of Mathematical Physics, 2 (1961) 212.

[3] A. M. Brodskii, D. Ivanenko, H. A. Sokolik, A new conception of the gravitational field, Zhurnal Eksp. Teor. Fiz., 41 (1961) 1307; Acta Phys. Hungar., 14 (1962) 21. 
[4] D.W. Sciama, On the analogy between charge and spin in general relativity, in Recent Developments in General Relativity, Festschrift for Infeld, Pergamon Press, Oxford; PWN, Warsaw (1962), pg. 415.

[5] F.W. Hehl, Four Lectures on Poincare Gauge Field Theories, in Cosmology and Gravitation, Plenum Press, New York (1980).

[6] K. Hayashi, T. Shirafuji, Gravity from Poincaré Gauge Theory of the Fundamental Particles, I IV, Progress of Theoretical Physics, 64 (1980), No. 3, 866; No. 3, 883; No. 4, 1435; No. 6, 2222.

[7] M. Blagojević, Gravitation and Gauge Symmetries, IOP Publishing, Bristol, 2002.

[8] A. Trautman, The Einstein-Cartan theory, in Encyclopedia of Mathematical Physics 2, J.-P. Francoise et al. (eds.), Elsevier, Oxford (2006) pg. 189.

[9] W. Kopczynski, A non-singular Universe with torsion, Physics Letters A, 39 (1972) 219.

[10] A. Trautman, Spin and Torsion can avert gravitational singularities, Nature (Phys. Sci.), 242 (1973) 7 .

[11] Nikodim J. Poplawski, Cosmology with torsion: an alternative to cosmic inflation, Physics Letters B, 694 (2010) 181.

[12] G.D. Kerlick, Bouncing" of simple cosmological models with torsion, Annals of Phys., 99 (1976) 127.

[13] J. Tafel, Cosmological models with a spinor field, Bull. Acad. Pol. Sci., Ser. math. astron. phys., 25 (1977) 593.

[14] Nikodim J. Poplawski, Nonsingular, big-bounce cosmology from spinor-torsion coupling, Phys. Rev. D, 85 (2012) 107502.

[15] A.V. Minkevich, Generalised cosmological Friedmann equations without gravitational singularity, Physics Letters A, 80 (1980) 232.

[16] A.V. Minkevich, Gauge approach to gravitation and regular Big Bang theory, Gravitation\&Cosmology 12 (2006) 11 [gr-qc/0506140].

[17] A.V. Minkevich, On gravitational repulsion effect at extreme conditions in gauge theories of gravity, Acta Physica Polonica B, 38 (2007) 61 [gr-qc/0512123].

[18] A.V. Minkevich and A.S. Garkun, Analysis of inflationary cosmological models in gauge theories of gravitation, Classical and Quantum Gravity, 23 (2006) 4237 [gr-qc/0512130].

[19] A.V. Minkevich, A.S. Garkun and V.I. Kudin, Regular accelerating universe without dark energy in Poincaré gauge theory of gravity, Classical and Quantum Gravity, 24 (2007) 5835 [arXiv:0706.1157].

[20] A.V. Minkevich, Gravitation, cosmology and space-time torsion, Annales de la Fondation Louis de Broglie 32 (2007) 253 [arXiv:0709.4337].

[21] A.V. Minkevich, Accelerating Universe with spacetime torsion but without dark matter and dark energy, Physics Letters B 678 (2009) 423 [arXiv:0902.2860].

[22] 2. A.S. Garkun, V.I. Kudin and A.V. Minkevich, Analysis of regular inflationary cosmological models with two torsion functions in Poincaré gauge theory of gravity, International Journal of Modern Physics A, 25 (2010) 2005 [arXiv:0811.1430].

[23] A.V. Minkevich, De Sitter spacetime with torsion as physical spacetime in the vacuum and isotropic cosmology, Modern Physics Letters A , 26 (2011) 259 [arXiv:1002.0538].

[24] A.V. Minkevich, Limiting energy density and a regular accelerating Universe in Riemann-Cartan spacetime, JETP Letters 94 (2011) 831.

[25] A.V. Minkevich, A.S. Garkun and V.I. Kudin, Relativistic cosmology and Poincaré gauge 
theory of gravity, in Einstein and Hilbert: Dark Matter, Editor: V. V. Dvoeglazov, Nova Science Publishers Inc (2011) 157.

[26] A.S. Garkun, V.I. Kudin, A.V. Minkevich and Yu.G. Vasilevsky, Numerical analysis of cosmological models for accelerating Universe in Poincare gauge theory of gravity, in Press, [arXiv:1107.1566].

[27] A.V. Minkevich, Gauge approach in gravitation theory, physical space-time and gravitational interaction, Space, time and fundamental interactions, issue 1 (2012) 62 (in rus.).

[28] A.V. Minkevich, To theory of regular accelerating Universe in Riemann-Cartan spacetime, in Press.

[29] F. Karakura, V.I. Kudin, A.V. Minkevich, About gravitational equations for homogeneous isotropic cosmological models with torsion, VINITI No 4512-82, Minsk (1982) 18 p. (in rus.)

[30] Dmitri Diakonov, Alexander G. Tumanov and Alexey A.Vladimirov, Low-energy General Relativity with torsion: a systematic derivative expansion, Phys. Rev.D , 84 (2011) 124042 [arXiv:1104.2432].

[31] P. Baekler, F.W. Hehl and J.M. Nester, Poincare gauge theory of gravity: Friedmann cosmology with even and odd parity modes. Analytic part, Phys. Rev.D , 83 (2011) 024001.

[32] V.I. Kudin, A.V. Minkevich, F.I. Fedorov, About space-time symmetries in gauge theory of gravity, VINITI No 3794-79, Minsk (1979) 12 p. (in rus.).

[33] M. Tsamparlis, Physics Letters A, 75 (1979) 27.

[34] A.V. Minkevich, A.S. Garkun, V.I. Kudin, Comment on "Torsion Cosmology and the Accelerating Universe" [arXiv:0811.1430]. 